\title{
A souped-up version of BCG
}

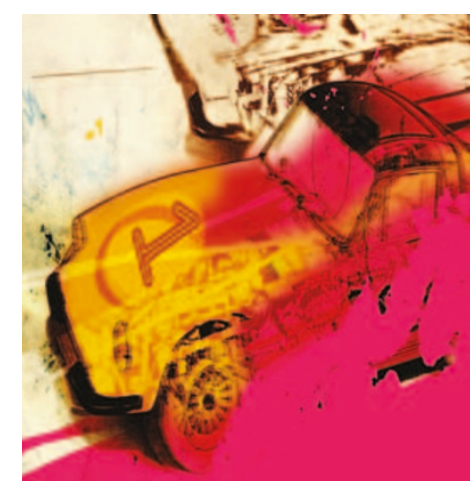

Mycobacterium bovis bacillus Calmette-Guérin (BCG) is a widely used vaccine against tuberculosis. It provides effective protection against childhood tuberculosis but is much less effective at protecting against adult disease. Now, Leander Grode and colleagues have shown that, compared with the parental strain of BCG, modified versions of BCG can provide increased protection against pulmonary tuberculosis in a mouse model.

Tuberculosis is no longer restricted to the developing world, and the emergence of novel genotypes, such as Mycobacterium tube rculosis W (also known as Beijing), that constitute a high proportion of drug-resistant strains has led to an urgent need to develop a more efficacious vaccine. Because of its location in the phagosome of cells, BCG is mainly processed and presented on MHC class II molecules, leading to preferential stimulation of $\mathrm{CD}^{+}{ }^{+} \mathrm{T}$ cells and weak stimula- tion of $\mathrm{CD}^{+} \mathrm{T}$ cells. However, it is $\mathrm{CD}^{+} \mathrm{T}$ cells that have been shown to be important in protective responses against $M$. tuberculosis. The authors reasoned that improving the ability of BCG to stimulate $\mathrm{CD} 8^{+} \mathrm{T}$-cell responses should result in a more effective vaccine.

The authors first developed two recombinant forms of BCG. The first strain, known as $h l y^{+}$rBCG, secretes the Listeria monocytogenes protein listeriolysin $\mathrm{O}$ (Hly), which allows escape from the phagosome. This strain was further modified to create a second strain, known as $\Delta u r e C$ $h l y^{+}$rBCG, which is deficient in urease $\mathrm{C}$, a molecule that has a role in $\mathrm{pH}$ neutralization of the phagosome. Deletion of the gene encoding urease $\mathrm{C}$ ensured an acidic $\mathrm{pH}$ in the phagosome, thereby promoting the activity of Hly.

$\mathrm{BALB} / \mathrm{c}$ mice were either not vaccinated or vaccinated intraperitoneally with a single dose of parental $\mathrm{BCG}, h l y^{+} \mathrm{rBCG}$ or $\Delta$ ureC $h l y^{+} \mathrm{rBCG}$, and these mice were challenged 120 days later with an aerosol of either a laboratory strain of $M$. tuberculosis $(\mathrm{H} 37 \mathrm{Rv})$ or the clinical isolate M. tuberculosis W. Although parental BCG and $h l y^{+}$rBCG reduced the M. tuberculosis load in the lungs of vaccinated mice compared with unvaccinated controls, $\Delta u r e C h l y^{+}$ rBCG provided considerably more protection, with a 1,000 -fold reduction in the M. tuberculosis load by 200 days after challenge. Similarly, although parental BCG provided almost no protection against the clinical isolate, $\Delta u r e C h l y^{+}$rBCG could reduce the $M$. tuberculosis load in lungs by 100-fold more than parental BCG.

The authors then analysed the distribution of mycobacterial antigens in infected cells. Macrophages infected with $\Delta u r e C h l y^{+}$rBCG had considerably more mycobacterial antigen in the cytoplasm than did cells infected with parental BCG. Similarly, cells infected with $\Delta u r e C h l y^{+}$rBCG underwent apoptosis, whereas cells infected with parental BCG showed little apoptosis. The authors propose that these results indicate the existence of two non-mutually exclusive mechanisms for the efficacy of $\Delta$ ure C $h l y^{+}$rBCG. First, Hly-mediated access to the cytosol allows loading of mycobacterial antigens onto MHC class I molecules. Second, release of phagolysosomal proteases into the cytosol leads to activation of cell-death pathways and induces apoptosis; apoptotic material is then taken up by antigen-presenting cells, leading to cross-priming.

Development of modified versions of BCG such as these could provide new ways of challenging the worldwide spread of tuberculosis. Clinical trials of $\Delta u r e C h l y^{+}$rBCG will commence in 2006.

(2) References and links ORIGINAL RESEARCH PAPER Grode, L. et al. Increased vaccine efficacy against tuberculosis of recombinant Mycobacterium bovis bacille Calmette-Guérin mutants that secrete listeriolysin. J. Clin. Invest. 115, 2472-2479 (2005) 